\title{
De novo mutations in SCN1A are associated (1) CrossMark with classic Rett syndrome: a case report
}

\author{
Mari Wold Henriksen ${ }^{1,2^{*}}$, Kirstine Ravn ${ }^{3 \dagger}$, Benedicte Paus ${ }^{2,4}$, Stephen von Tetzchner ${ }^{5}$ and Ola H Skjeldal ${ }^{6}$
}

\begin{abstract}
Background: Rett syndrome (RTT) is a neurodevelopmental disorder. In more than 95\% of females with classic RTT a pathogenic mutation in MECP2 has been identified. This leaves a small fraction of classic cases with other genetic causes. So far, there has not been reported any other gene that may account for the majority of these cases.

Case presentation: We describe two females who fulfill the diagnostic criteria for classic RTT, with pathogenic de novo mutations in SCN1A, which usually leads to Dravet syndrome. The developmental history and clinical features of these two females fits well with RTT, but they do have an unusual epileptic profile with early onset of seizures. Investigation of mRNA from one of the females showed a significantly reduced level of MECP2 mRNA.

Conclusions: To our knowledge, this is the first report suggesting that SCN1A mutations could account for a proportion of the females with classic RTT without MECP2 mutations. As a consequence of these findings SCN1A should be considered in the molecular routine screening in MECP2-negative individuals with RTT and early onset epilepsy.
\end{abstract}

Keywords: Rett syndrome, Epilepsy, Genetics, SCN1A, Dravet syndrome

\section{Background}

Rett syndrome (RTT, OMIM 312750) is a severe neurodevelopmental disorder, characterized by an apparently normal development the first 6-18 months, followed by regressive loss of acquired skills [1]. The current diagnostic criteria for classic RTT require a period of regression, loss of acquired purposeful hand skills and acquired spoken language (if any), gait abnormalities and stereotypic hand movements. Exclusion criteria include grossly abnormal psychomotor development in the first 6 months of life or known brain injury [1]. In more than $95 \%$ of females with classic and $50 \%$ with atypical RTT, a pathogenic mutation in $M E C P 2$ has been identified [1]. Mutations in 69 other genes have in recent years been associated with RTT and RTT-like disorders [2, 3], including a girl with a RTT-like condition and a mutation in SCN1A [4]. The present study reports two females fulfilling the diagnostic criteria for classic RTT [1] with de novo

\footnotetext{
* Correspondence: mari.wold.henriksen@vestreviken.no

${ }^{+}$Mari Wold Henriksen and Kirstine Ravn contributed equally to this work. 'Department of Neurology, Vestre Viken Hospital Trust, Drammen Hospital, P.O. Box 800, 3004 Drammen, Norway

${ }^{2}$ Institute of Clinical Medicine, Faculty of Medicine, University of Oslo, P.O.

Box 1171, Blindern, 0318 Oslo, Norway

Full list of author information is available at the end of the article
}

mutations in SCN1A. Pathogenic mutations in SCN1A are known to cause Dravet syndrome [5] and have not to our knowledge been associated with classic Rett syndrome.

\section{Case presentations}

Case 1

Case 1 is a 19 years old woman (for timeline see Fig. 1). She was born at 37 weeks gestation with a birth weight of $2890 \mathrm{~g}$, length $47 \mathrm{~cm}$, and a head circumference of $32 \mathrm{~cm}$. Pre- and neonatal periods were normal. She had her first seizure, a prolonged febrile seizure, at 5 months of age. She developed afebrile focal seizures and intractable generalized seizures, both myotonic, tonic and tonic-clonic. She has had several episodes with convulsive status epilepticus. Her early development, however, was unremarkable. She developed normal hand function, including a pincer grip, and started to use a few words, 15 at the most. She began walking independently at 17 months. However, from around 15 months of age her development slowed down and she gradually lost acquired skills. She stopped using her hands, her words disappeared and her gait became broad-based and ataxic. She developed midline rubbing hand stereotypies, although not very intense, and bruxism. She often had breath-holding spells and infrequently she hyperventilated.

(c) The Author(s). 2018 Open Access This article is distributed under the terms of the Creative Commons Attribution 4.0 International License (http://creativecommons.org/licenses/by/4.0/), which permits unrestricted use, distribution, and 


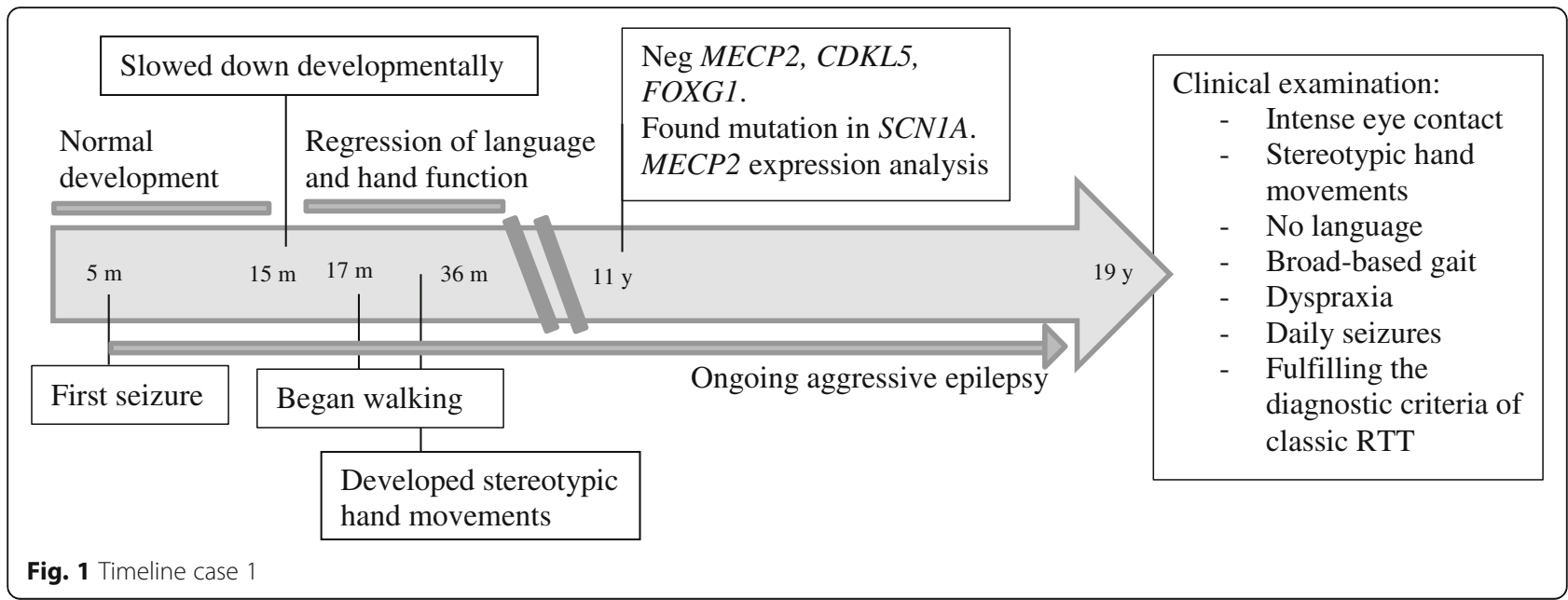

Her sleep pattern was impaired with night time screaming spells and occasionally laughing spells. Between one and 2 years of age, she developed autistic traits. She had a deceleration of head growth from 50th to 10th percentile.

The clinical examination at 19 years revealed a woman with intense eye contact and ongoing stereotypic hand movements with hand dyspraxia. She had a broad-based gait with notable ataxia. Breath holding and teeth grinding were observed. She was only $141 \mathrm{~cm}$ tall, but had normal weight for height. Her musculature was generally hypotonic and she had a slight scoliosis. Her epilepsy was still aggressive with daily seizures (focal, tonic and tonicclonic), despite intense anti-epileptic treatment. Her clinical signs and symptoms were consistent with classic RTT, fulfilling the criteria of this disorder.

$\mathrm{CT}$ and MRI scans of the brain were unremarkable. At the age of eleven MECP2, CDKL5, and FOXG1 were analyzed with Sanger sequencing of all exons with flanking intron regions and MLPA kits P015C, P395 and P189 from MRC-Holland, all with normal results. Due to the aggressive epilepsy SCN1A was Sanger sequenced and this disclosed the novel splice variant NG_011906.1:g.76169G > C, (NM_001165963.2): c.4284+1G >C. Using Alamut Visual software (Interactive Biosoftware, France) and the guidelines of American College of Medical Genetics and Genomics and the association for Molecular Pathology (ACMG) [6], this variant was scored as pathogenic. Parental testing indicated that the mutation was de novo. Two splice mutations (c.4284 +1G > T and c. $4284+1 \mathrm{G}>\mathrm{A}$ ) affecting the same splice site, have previously been reported in Dravet syndrome [7, 8]. Because she fulfilled the criteria for RTT, but no mutation in $M E C P 2$ was identified, a $M E C P 2$ gene expression analysis, performed on mRNA isolated from her fibroblasts was performed. This analysis indicated that her $M E C P 2$ expression level was more than $80 \%$ reduced compared to three female controls (Fig. 2).

\section{Case 2}

Case 2 is a 32 years old woman (for timeline see Fig. 3). She was born at 40 weeks of gestation with a birth weight of $3830 \mathrm{~g}$, length $52 \mathrm{~cm}$, and a head circumference of $36 \mathrm{~cm}$. Pre- and neonatal periods were normal. At 7 months, she had her first seizure, a febrile bilateral tonic-clonic seizure. Between one and 2 years of age her epilepsy became more severe, with daily generalized seizures. The frequency of seizures declined somewhat when she reached school age, but her epilepsy remained drug resistant, with several bilateral tonic-clonic seizures every week. Besides the epilepsy, her development was apparently normal the first $12-15$ months. She sat independently at 7 months. At 1 year, she used a few words and had an appropriate use of hands. She learned to walk when she was 24 months old. When she was between 12 to 15 months of age she started to lose acquired skills. Her hand function deteriorated gradually, her words disappeared and she no longer seemed to show interest in her surroundings. She developed bruxism and hand-washing stereotypies. She could walk independently until school age, but then she gradually needed support when walking. Through childhood her sleep pattern was significantly disturbed with both screaming and laughing spells. Her respiration has however never been affected.

The clinical examination revealed a 32 years old woman who could walk a few meters with support, had ataxic and apraxic hand movements, but not hand stereotypies. She had no language but gave intense eye contact. Her muscle tone was normal. She had a slight scoliosis. Her epilepsy was still a major concern, with daily to weekly bilateral tonic-clonic seizures. She fulfilled the criteria of classic RTT.

Genetic analyses of $M E C P 2$ at the age of 18 gave negative results (Sanger sequencing and MLPA kit P015 from 


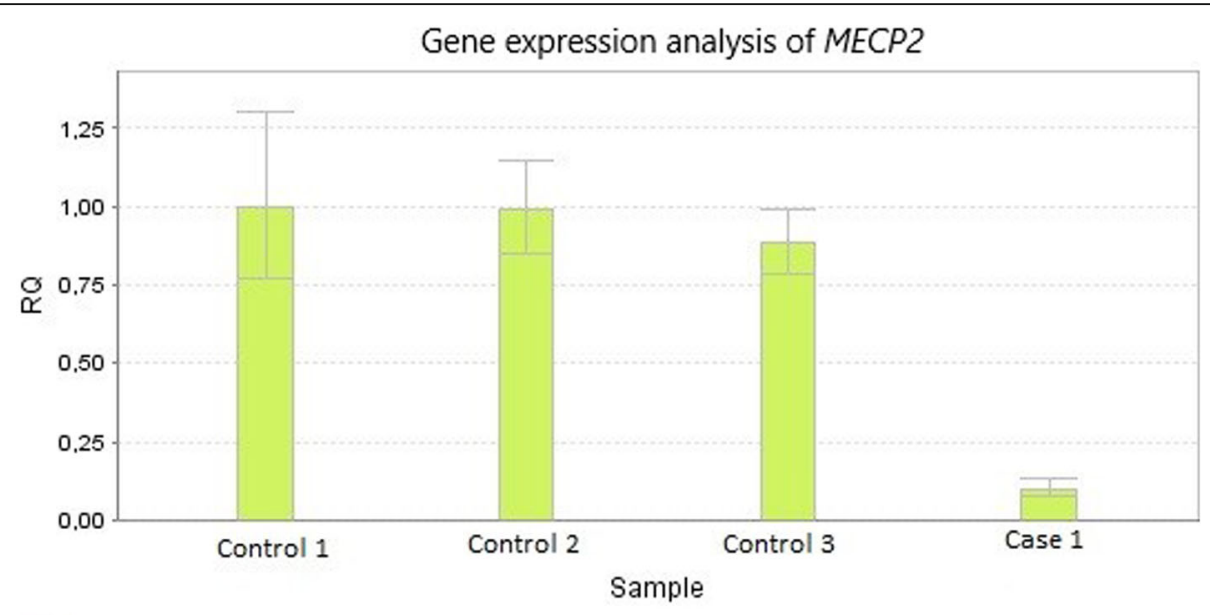

MECP2_1

Fig. 2 The relative expression level of MECP2 (the alternative transcripts coding for isoforms MeCP2_E1) in cultured fibroblasts of three female controls and Case 1. The MECP2 gene expression analysis was performed on mRNA isolated from her fibroblasts, using Applied Biosystems 7500 Fast Real-Time PCR System (ABI, Foster City, CA), with pre-designed assay (TaqMan MGB probe HS01598237). GAPDH served as an endogenous control. The RQmin and RQmax with the confidence interval set at 95\%:Female1; RQ Min 0.77, RQ Max 1.29, Female2; RQ Min 0.85, RQ Max 1.14, Female3; RQ Min 0.787, RQ Max 0,99, Patient; RQ Min 0.07, RQ Max 0,13

MRC-Holland). As a participant in a national survey of females with RTT she was recently retested by applying whole exome sequencing (WES) using Agilent SureSelect Target Enrichment Kit (Agilent Technologies, Santa Clara, CA) on Illumina HiSeq 2500 with pair-end runs. Alignment, mapping, and variant calling were performed using Genome Analysis Tool Kit (GATK). Reads were mapped to the reference sequence (GRCh37/hg19). Following bioinformatic filtration, analysis of coding regions and intron/exon boundaries of 1479 predefined genes (including FOXG1, CDKL5 and SCN1A with a $100 \%$ coverage at a depth $>10 \times$ ) was performed. WES disclosed the variant, NG_011906.1:g.76130G > T, NM_001165963.1: c.4246 G > T, p.(Asp1416Tyr) in SCN1A. Using Alamut Visual software (Interactive Biosoftware, France) and ACMG criteria [6] this novel variant was scored as pathogenic. Parental testing indicated that the mutation was de novo. This is a novel variant, but mutations affecting the same amino acid have been reported in Dravet syndrome [9].

\section{Discussion and conclusions}

We present two females with clinical pictures consistent with classic RTT and who fulfill the diagnostic criteria for this disorder [1], but without mutations in the coding regions of

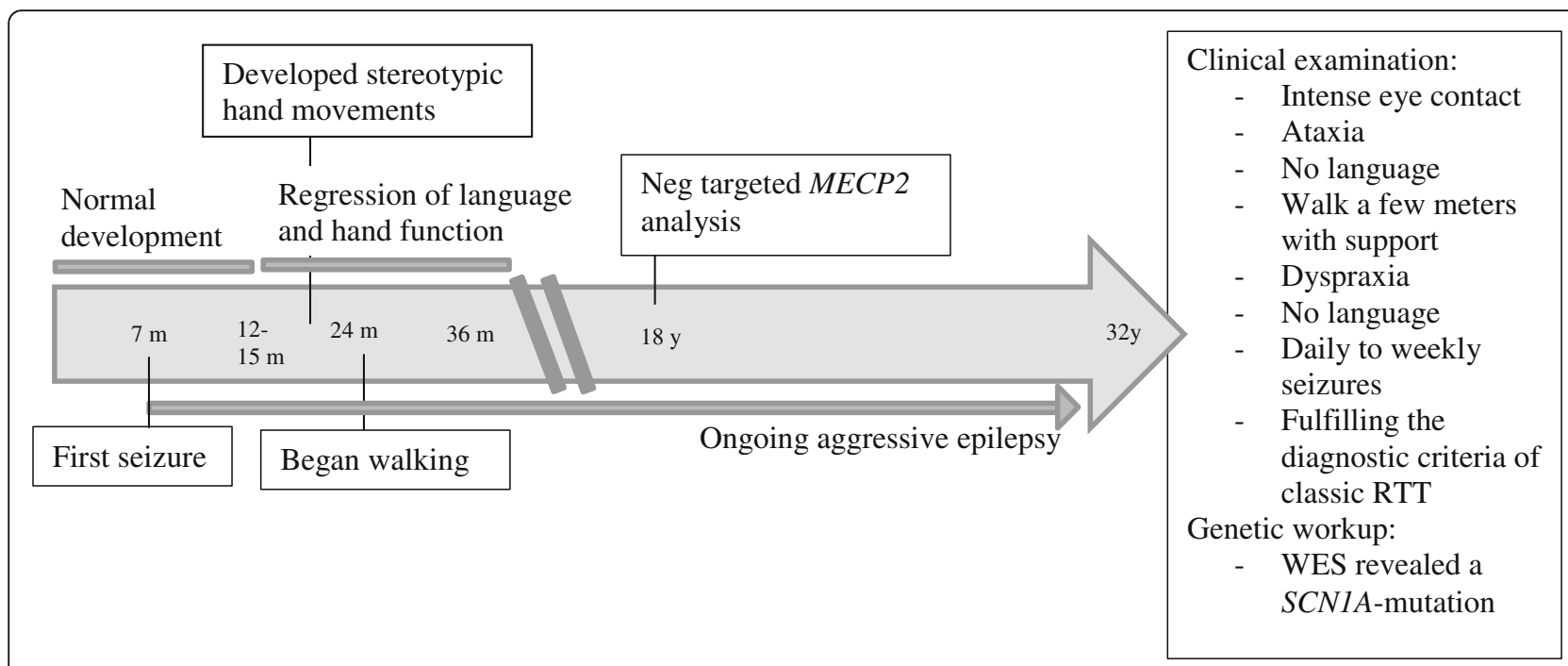

Fig. 3 Timeline case 2 
$M E C P 2, C D K L 5$ and FOXG1. However, deep intronic mutations and duplications/deletions of exons not covered by the MLPA analysis, have not been excluded.

Further genetic analyses revealed presumed pathogenic de novo mutations in $S C N 1 A$ in both. More than $80 \%$ of individuals with pathogenic mutations in SCN1A have Dravet syndrome [10]. Both females do have clinical features associated with this syndrome, like early seizure onset, prolonged febrile seizures, status epilepticus, and drug resistant epilepsy [5]. Dravet syndrome has no clearly defined diagnostic criteria and the phenotypic spectrum is wide. These case reports show that there may be a clinical overlap between features of RTT and other neurodevelopmental disorders, such as Dravet syndrome. This is a challenge for disease classification and diagnosis. Strict and robust criteria are necessary for making consistent diagnoses and sorting out differential diagnosis. Recognizing potential confusion, the revised RTT criteria suggest specifying both phenotype and mutation [1].

Finding the molecular basis is important in clinical practice, for prognosis and genetic counseling, and it may have implications for treatment. It may also be essential for better understanding of the pathophysiology. For instance, in Case 1, harboring the SCN1A splice site mutation, quantitative gene expression analyses showed a reduced level of $M E C P 2$ mRNA in fibroblasts, although no MECP2 mutation was detected. In order to evaluate the significance of this finding further research is demanded. Both females presented here participated in a national survey of the Norwegian population of females with RTT. This survey includes 93 participants with RTT and RTT-like disorders, 74 with classic RTT. A total of 12 participants did not have mutations in $M E C P 2$, three in the group with classic RTT, including the two females presented here $(2.7 \%$ of the participants with classic RTT in this cohort). The presence of these two cases in the Norwegian RTT cohort indicates that SCN1A mutations could account for a significant part of the population of females with classic RTT without MECP2 mutations. Although fulfilling the diagnostic criteria for classic RTT their epileptic profile is atypical with early seizure onset and prolonged febrile seizures. The possibility that the two females' phenotype might be a result of two mutations, one $S C N 1 A$ and one rare intronic variation in $M E C P 2$ or $C D K L 5$, seems unlikely with our present knowledge.

In the cohort of 74 individuals with classic RTT these two individuals and two others were the only ones with seizure onset before regression. The findings in this paper could lead to justifying the inclusion of $S C N 1 A$ in the molecular routine screening for $M E C P 2$-negative individuals with RTT and early onset epilepsy.

\section{Abbreviations}

ACMG guidelines: Guidelines of American College of Medical Genetics and Genomics and the association for Molecular Pathology; GATK: Genome
Analysis Tool Kit; MLPA: Multiplex Ligation-dependent Probe Amplification; RTT: Rett syndrome; WES: Whole Exom Sequencing

\section{Acknowledgements}

We would like to thank the two women and their families for participating, and to Hilde Breck for help with collecting data.

\section{Funding}

MWH is funded by Vestre Viken Hospital Trust. Vestre Viken Hospital Trust has not had any role in the design of the study, data collection, analysis, interpretation of data or in writing the article.

\section{Availability of data and materials}

The datasets used and analyzed during the current study are available from the corresponding author on reasonable request.

\section{Authors' contributions}

$\mathrm{MWH}$ collected clinical information and was a major contributor to the writing of the manuscript. KR conducted the MECP2 gene expression analysis and was a major contributor to the writing of the manuscript. OHS led and supervised the project, contributed in writing and interpretation. SVT and BP contributed with interpretation of data and critical reviews of the article. All authors read and approved the final manuscript.

\section{Ethics approval and consent to participate}

Informed parental consent was obtained for both females. Ethics approval was obtained from the Regional Committee for Medical Research Ethics, South East Norway (ethical agreement no. 2012/1572).

\section{Consent for publication}

Written informed consent for publication of their clinical details was obtained from the parents of the patients. A copy of the consent form is available for review by the Editor of this journal.

\section{Competing interests}

The authors declare that they have no competing interests.

\section{Publisher's Note}

Springer Nature remains neutral with regard to jurisdictional claims in published maps and institutional affiliations.

\section{Author details}

${ }^{1}$ Department of Neurology, Vestre Viken Hospital Trust, Drammen Hospital, P.O. Box 800, 3004 Drammen, Norway. ${ }^{2}$ Institute of Clinical Medicine, Faculty of Medicine, University of Oslo, P.O. Box 1171, Blindern, 0318 Oslo, Norway. ${ }^{3}$ Department of Clinical Genetics, Rigshospitalet, University of Copenhagen, Blegdamsvej 9, 2100 København $\varnothing$, Copenhagen, Denmark. ${ }^{4}$ Department of Medical Genetics, Oslo University Hospital, P.O. Box 4950, 0424 Oslo, Norway. ${ }^{5}$ Department of Psychology, University of Oslo, P.O. Box 1094, Blindern, 0317 Oslo, Norway. ${ }^{6}$ Gillberg Neuropsychiatric Centre, Sahlgrenska University of Gothenburg, Kungsgatan 12, 41119 Gothenburg, Sweden.

Received: 1 June 2018 Accepted: 27 September 2018

Published online: 11 October 2018

\section{References}

1. Neul JL, Kaufmann WE, Glaze DG, Christodoulou J, Clarke AJ, Bahi-Buisson N, Leonard H, Bailey ME, Schanen NC, Zappella M, et al. Rett syndrome: revised diagnostic criteria and nomenclature. Ann Neurol. 2010;68(6):944-50.

2. Ehrhart F, Sangani NB, Curfs LMG. Current developments in the genetics of Rett and Rett-like syndrome. Curr Opin Psychiatry. 2018;31(2):103-8.

3. Percy AK, Lane J, Annese F, Warren H, Skinner SA, Neul JL. When Rett syndrome is due to genes other than MECP2. Transl Sci Rare Dis. 2018;3(1):49-53.

4. Lucariello M, Vidal E, Vidal S, Saez M, Roa L, Huertas D, Pineda M, Dalfo E, Dopazo J, Jurado P, et al. Whole exome sequencing of Rett syndrome-like patients reveals the mutational diversity of the clinical phenotype. Hum Genet. 2016;135(12):1343-54.

5. Dravet C. The core Dravet syndrome phenotype. Epilepsia. 2011;52(Suppl 2):3-9.

6. Richards S, Aziz N, Bale S, Bick D, Das S, Gastier-Foster J, Grody WW, Hegde M, Lyon E, Spector E, et al. Standards and guidelines for the interpretation of sequence variants: a joint consensus recommendation of the American 
College of Medical Genetics and Genomics and the Association for Molecular Pathology. Genet Med. 2015;17(5):405-24.

7. Kwong AK, Fung CW, Chan SY, Wong VC. Identification of SCN1A and PCDH19 mutations in Chinese children with Dravet syndrome. PLoS One. 2012;7(7):e41802.

8. Zuberi SM, Brunklaus A, Birch R, Reavey E, Duncan J, Forbes GH. Genotypephenotype associations in SCN1A-related epilepsies. Neurology. 2011;76(7): 594-600.

9. Depienne C, Trouillard O, Saint-Martin C, Gourfinkel-An I, Bouteiller D, Carpentier W, Keren B, Abert B, Gautier A, Baulac S, et al. Spectrum of SCN1A gene mutations associated with Dravet syndrome: analysis of 333 patients. J Med Genet. 2009;46(3):183-91.

10. Meng H, Xu HQ, Yu L, Lin GW, He N, Su T, Shi YW, Li B, Wang J, Liu XR, et al. The SCN1A mutation database: updating information and analysis of the relationships among genotype, functional alteration, and phenotype. Hum Mutat. 2015;36(6):573-80.

Ready to submit your research? Choose BMC and benefit from:

- fast, convenient online submission

- thorough peer review by experienced researchers in your field

- rapid publication on acceptance

- support for research data, including large and complex data types

- gold Open Access which fosters wider collaboration and increased citations

- maximum visibility for your research: over $100 \mathrm{M}$ website views per year

At BMC, research is always in progress.

Learn more biomedcentral.com/submissions 